\title{
Une marge de la psychopathologie contemporaine : les « enfants indigo »
}

\author{
Renaud Evrard et Pascal le Maléfan
}

Les «enfants indigo » est le nom générique donné par un ensemble de mouvements New Age à des enfants considérés comme «surhumains » et qui se présentent généralement avec des difficultés censées découler de l'inadaptation de leur environnement à leur supposé haut potentiel. En gestation depuis les années 1970, le mouvement s'est vraiment formalisé dans les années 1990, et se répand dans les pays francophones depuis 1999. Les organismes de lutte anti-sectes ont déjà répertorié les nombreux dangers accompagnant ce discours. Cet article n'est pas le lieu pour rappeler ces conseils de prudence, même si quelques références seront données sur ce point. L'intérêt de ce travail est de produire une description plus générale des conditions d'émergence et de développement des mouvements autour des « enfants indigo », de façon à situer leur «niche écologique ». Cette description sera utile aux approches psychopathologiques infanto-juvéniles car ces références culturelles pénètrent le champ de la santé mentale, ainsi que l'illustrera le cas clinique de Victor.

\section{Façonner un enfant indigo}

Le concept d'«enfant indigo » a été inventé par Nancy Ann Tappe, une voyante américaine affirmant observer des «auras» de différentes couleurs autour de certaines personnes, dont la couleur indigo. Elle reprend alors un thème classique de l'ésotérisme, ouvrant un champ très large de différents profils psychospirituels basés sur le type d'aura. À partir d'un best-seller dû à des «écrivains-thérapeutes » comme le New Age en connaît 
beaucoup - Les Enfants indigo, enfants du troisième millénaire [6], écrit par Lee Carroll et Jan Tober -, l'idée s'est répandue que des «enfants indigo », des enfants «mutants », nés depuis les années 1990, surdoués et rebelles, seraient parmi nous pour nous aider à affronter la « grande transition planétaire ». Des parents sont alors amenés à voir dans le comportement « étrange »- à leurs yeux - de leur enfant et souvent de leur adolescent, le signe d'aptitudes spirituelles et paranormales particulières.

Ces affirmations sont certes portées par une sorte de millénarisme, mais elles font écho à des réalités sociales et culturelles qui ne peuvent désintéresser les psychopathologues. Tout d'abord, Lee Carroll se prétend contacté par une entité spirituelle, Kryeon, un ange annonciateur de l'apocalypse et de sa solution : l'accueil des enfants indigo. Il y a donc une source tout à fait sectaire ${ }^{1}$, ainsi qu'un marché du livre New Age lucratif accompagnant ce mouvement. Mais il existe surtout une convergence étonnante entre cette mise en avant des «enfants indigo » et le diagnostic de TDAH (Trouble de déficit de l'attention / hyperactivité $)^{2}$. Celui-ci est généralement associé à la prescription de Ritaline $®$, un stimulant ayant des effets paradoxaux sur les personnes hyperactives, puisqu'il calme l'agitation et favorise l'attention. Diagnostics et prescriptions ont subi une inflation très importante outreAtlantique depuis les années 1990, et progressivement dans d'autres pays occidentaux. Ils occasionneraient une perception nouvelle et pessimiste de la jeunesse, dont la réussite dans la vie dépendrait de ces formes de soutiens biochimiques. Certaines personnes pensent assister à une explosion inquiétante de la demande de ce médicament pour des raisons extrathérapeutiques, notamment sous l'insistance des parents ou à la demande des enseignants. Pour d'autres, cette demande croissante s'explique par une meilleure connaissance de la maladie et donc un meilleur diagnostic. Quelque soit l'issue de cette controverse, la Ritaline ${ }^{\circledR}$

\footnotetext{
$1 \quad$ Ce groupement est qualifié de secte en France mais pas aux États-Unis. Son point fort est que les adeptes ne possèdent pour objets de culte que leurs propres enfants, ce dont ils ne peuvent se débarrasser, cf. [11, p. 13].

Ce syndrome est caractérisé par des états d'hyperactivité, des moments d'absence, des changements d'humeur, des problèmes de concentration et d'impulsivité.
} 
a glissé dans ce cliché du médicament comme réponse sociale, soutenue par une biopolitique répressive. Or la description quasi clinique des enfants indigo par leurs promoteurs recoupe largement celle conduisant au diagnostic de TDAH. Tant et si bien que l'on peut donner une double description du même enfant, l'une offrant des promesses plus porteuses sur le plan narcissique. Le même type de recouvrement permet de recatégoriser des enfants en échec scolaire, autistes, dyslexiques, des «ados en crise», voire même n'importe quel comportement trivial d'un enfant, comme la frustration, l'impatience ou la bouderie. Russell Barkley, professeur de psychiatrie à New York, rappelle que ces check-lists donnant une description vague et générique de ce que serait un enfant indigo sont facilement validées subjectivement, selon un effet psychologique connu sous le nom d'effet Barnum-Forer [21]. Les traits attribués aux enfants indigo pourraient en réalité décrire n'importe qui.

Une autre convergence de taille est celle avec les surdoués ou à haut potentiel. Sophie Cote, présidente de l'Association Française des Enfants Précoces (AFEP), et Brigitte Bernadet, présidente de l'Association Nationale Pour les Enfants Intellectuellement Précoces (ANPEIP), constatent que les descriptions des enfants indigo recouvrent celles des enfants précoces [9, 19]. Ce chevauchement serait voulu par les tenants des indigos, même s'il est perceptible qu'une dynamique similaire rapproche les deux groupes : les parents font de plus en plus la démarche de venir demander d'évaluer le QI de leurs enfants probablement surdoués ou précoces, puisqu'en difficulté à l'école ou incontrôlables à la maison. C'est la même espérance, et la même plainte à propos d'enseignants et d'éducateurs qui ne traitent pas l'enfant avec tous les égards qu'il mérite.

Le thème de l'inquiétante étrangeté de l'enfance est porteur. «Ces enfants, qualifiés d'indigo, sont en fait une nouvelle version de ces "enfants nouveaux" qui, depuis un certain nombre d'années, semblent cristalliser tous les espoirs et les angoisses du monde des adultes et désigner l'enfance comme le lieu d'une irréductible et problématique altérité » [31, p. 138]. 
Un exemple en est « l'enfant téflon » inventé par le psychologue québécois Daniel Kemp [20] dont l'opposition blindée à notre société dissimulerait une promesse d'avenir. Ces enfants «nouveaux » (par rapport aux enfants « anciens » qui, eux, peuvent s'adapter aux structures en place) voguent entre le psychologique normal, le pathologique et le merveilleux.

L'intérêt des tenants d'un «syndrome indigo » est manifestement de mettre ces enfants ou adolescents sur le même plan que les surdoués incompris, les génies artistiques, les penseurs révolutionnaires en herbe, les humanistes ou les mystiques. Les mouvements des enfants indigo leur injectent un nouveau potentiel, pouvant se révéler dans un certain contexte éducatif, par exemple la scolarisation à domicile ou les écoles spécialisées (Montessori, Steiner, Freinet). Ce mythe contemporain jouerait le même rôle que l'appellation de « l'enfantancêtre », dans l'Afrique de l'Ouest, enfant dont le comportement est différent de celui des autres, soit en négatif, soit en positif, parce que son esprit est occupé par un ancêtre mort [27]. Cette injection de sens dans le devenir de l'enfant (et sa perception par le parent) est un des moteurs des mouvements indigo, malgré toutes les critiques possibles de sa mise en forme.

Suite au premier livre, des parents ont reconnu leurs enfants comme étant « indigo » et des adolescents ou adultes se sont identifiés eux-mêmes comme tels. Le second ouvrage de Carroll et Tober - Célébration des enfants indigo [7] - est d'ailleurs une compilation de témoignages, de récits et d'anecdotes transmis par les lecteurs du premier livre. Des dizaines d'ouvrages sur le sujet ont, depuis, été publiés et traduits. La structure même des livres exprime l'ésotérisme contemporain : renvois constants vers Internet, pour une activité en réseau et participative, où on se bricole une croyance à la carte. Ainsi, le lecteur est incité à sauter un chapitre du premier livre [6] si celui-ci risque d'aller à l'encontre de sa religion occidentale (cf. [31, p. 133-138]). Et cela fonctionne : la communauté Internet est gigantesque et des thérapeutes se regroupent autour du label de l'EMF-Balancing, technique d' «harmonisation des champs électromagnétiques ». Mais il est difficile de chiffrer le 
nombre d'adeptes, comme l'explique la Miviludes, Mission interministérielle de vigilance et de lutte contre les dérives sectaires [19]: les mouvements New Age sont plus difficiles à cerner que les sectes des années 1970, car la notion de groupe a disparu, et il ne reste plus qu'un melting-pot de croyances protéiformes, évolutives et faites sur-mesure.

La France semble se montrer progressivement réceptive à ce mouvement. Plusieurs analyses critiques du mouvement indigo circulent déjà en français ${ }^{3}$, ainsi que de bonnes analyses sociologiques [31, pp. 129-177]. Mais on peut se demander quels sont les motifs psychologiques sous-jacents à la mise en place de ces discours non sans effets sur la clinique, en ce qu'ils viennent interroger l'incompréhension des générations et des formes contemporaines de la psychopathologie. Ils révèlent également les méfaits possibles d'une médicalisation de l'enfance et de l'adolescence, d'une sur-thérapeutisation entrant dans une économie de marché. Le phénomène des indigo serait donc à double face.

\section{Un effet délétère de la psychopathologisation de l'enfance?}

Comme l'écrit le psychanalyste Norbert Bon [3] : «il n'est pas nouveau que des parents se saisissent ainsi d'un signifiant venant connoter positivement les difficultés de leur enfant et, à cet égard, ces auteurs [les tenants du syndrome indigo] ne manquent pas de fustiger, à juste titre, l'inflation des diagnostics péjoratifs d'ADD (attention deficit desorder) ou d'ADHD (hyperactivité), qui viennent figer dans un dysfonctionnement neurobiologique les difficultés de l'enfant. (...) Pour le reste, dès que quelqu'un souffre ou se trouve dans le désarroi, il se trouve un prophète pour lui fournir le signifiant qui dans l'Autre orchestrerait sa jouissance et parfaire ainsi sa méconnaissance. » La position du psychopathologue est donc

\footnotetext{
3 Deux travaux d'étudiants en Zététique et approche scientifique du paranormal sont disponibles sur
} http://esprit.critique.free.fr/ 
intermédiaire : il admet les problèmes posés par la psychopathologisation de l'enfance et de l'adolescence, mais il semble avoir du mal à évaluer la réponse sociale qui en découle. Cependant, il est possible d'envisager que les deux soient liées, l'émergence des «enfants indigo » devenant une réaction aux données contemporaines de la psychopathologie. C'est aussi à ce titre que ce thème doit intéresser les cliniciens.

L'industrie pharmaceutique Novartis, qui fabrique la Ritaline ${ }^{\circledR}$, est au cœur d'une controverse quant à son intervention présumée en faveur d'une hypermédicalisation de l'enfance et de l'adolescence, afin d'écouler ses produits. Le phénomène hante l'imaginaire social, après quelques constats sur le lien ténu entre pharmaceutique, nosologie et économie. Il existe en effet une très grande disparité des chiffres de prévalence de ce syndrome selon les pays ou l'époque étudiée, variant entre moins de $1 \%$ (Hong-Kong) jusqu'à $25 \%$ (États-Unis). L'explication de ces disparités n'est pas claire : critères différents, facteurs culturels ? Il faut dire que les psychologues américains sont habilités à faire le travail de prescription réservé, en Europe, aux pédopsychiatres. Swanson et al. [30] soulignent en 1998 que cette prévalence semble parfois être corrélée à l'agressivité de la politique marketing autour de la Ritaline ${ }^{\circledR}$, conçu comme le principal traitement. En cinq ans, le chiffre des ventes a été multiplié par 40 : 0,7 million de cachets en 1995, 31 millions en 1999. Le néo-libéralisme post-moderne pousse justement à faire de la santé en général, et de la santé mentale en particulier, un ensemble de marchés à conquérir [15]. Les acteurs du champ de la psychopathologie s'étonnent, et parfois s'alertent, de la construction de nouveaux diagnostics pour des raisons majoritairement extrascientifiques [28].

Le philosophe canadien Ian Hacking a donné ses lettres de noblesse à l'étude de ces mutations des catégories dans les sciences humaines et sociales. Il a repéré notamment des «maladies mentales transitoires » qui vont subitement être de plus en plus diagnostiquées pendant une période avant de quasiment disparaître, à la fois sur la carte nosographique et 
dans le territoire psychopathologique. Parmi les exemples, la monomanie [14], les délires médiumniques [22], les troubles de personnalité multiples [16]. Pour décrire le cadre complexe de facteurs en jeu, Hacking utilise la métaphore de la «niche écologique » [17], c'est-à-dire l'ensemble des conditions sociologiques, philosophiques, économiques... qui permettent à une catégorie de prospérer malgré une compétition avec d'autres.

Même si cette description reste une métaphore issue de la théorie de l'évolution en biologie, elle essaye de rendre compte d'une certaine efficacité. Suivant un mécanisme d'interaction et de boucle rétroactive, ce qui peut être perçu comme une construction de l'esprit peut concrètement «façonner les gens » et les conduire à identifier leurs problèmes avec la description, temporairement plus adaptée, qu'on leur propose. Ainsi, de ce point de vue, les enfants indigo existent vraiment. Quelque soit l'artificialité de la catégorie qui les englobe, les enfants indigo ne sont pas des artefacts : ils sont des êtres humains adaptés à une niche écologique. Ce constat est important car il doit conditionner la réaction du psychopathologue: plutôt que de nier en bloc l'existence de cette catégorie, il se doit d'apprendre à connaître sa niche écologique. Cela pourra le préserver de simplement grossir le rang des opposants, renforçant la frontière extérieure de ce discours, et par-là même l'efficacité d'une doctrine basée sur une révélation opprimée. Il pourrait alors «négocier» une thérapeutique adaptée.

Mais si la niche écologique des enfants indigo a accompagné l'inflation du diagnostic de TDAH aux États-Unis, les mêmes conditions infiltrent les systèmes de références européens, en témoignent les controverses en France autour de la prévention de la délinquance axée sur la détection de troubles du comportement dès la naissance, ou encore l'aura actuelle des troubles de l'apprentissage et autres "dysfonctions ». L'inflation de la psychopathologisation de l'enfance renvoie, sur le plan de l'imaginaire social, au mythe d'une psychiatrie répressive privilégiant son rôle d'hygiène mentale au service du plus puissant. 
Cela excite la production de réponses révoltées, prenant parfois des voies marginales. Tels des parasites [17, p. 182], les mouvements des enfants indigo se greffent sur les arguments des tenants d'une reconsidération de la psychopathologisation et de la médicalisation de l'enfance et de l'adolescence. Ces parasites jouent alors un rôle lors de ce flottement de notre vision anthropologique de la jeunesse.

\section{Victor, enfant doré et adoré ?}

Victor, 7 ans, est décrit comme un «enfant doré », autre appellation pour l'enfant « indigo ». En effet, il aurait des « dons », notamment celui de guérir et celui de communiquer par télépathie. Mais sa mère a encore du mal avec cette catégorisation qu'elle a trouvé sur Internet. Elle souhaiterait que son enfant « reste normal ». De la même manière qu'elle ne veut pas qu'on teste son QI, alors que la maîtresse le dit brillant, elle ne veut pas qu'il soit un cobaye des parapsychologues. Si elle contacte le Service d'Orientation et de Soutien des Personnes Sensibles aux Expériences Exceptionnelles, un espace clinique développé par la plus ancienne fondation consacrée à la recherche en parapsychologie en France, l'Institut Métapsychique International (IMI), c'est qu'elle cherche quelqu'un capable d'entendre cette paranormalité. Elle est reçue par le premier auteur (RE), l'un des deux psychologues engagés alors dans ce service.

Lors du premier entretien, elle explique qu'elle a trouvé l'IMI grâce à une amie belge elle-même médium. Cette amie serait en contact télépathique permanent avec Victor : un jour, les deux femmes étaient tellement prises dans une conversation téléphonique que la mère de Victor allait arriver en retard à l'école. L'amie belge proposa d'envoyer un message télépathique pour le prévenir. Ensuite, lorsque sa mère l'appela, Victor aurait tout de suite expliqué qu'il avait été « mis au courant » par l'amie belge. 
Le psychologue est donc d'emblée mis en face du « fait paranormal » et de sa réaction va dépendre la suite de la prise en charge. Car s'il se contente d'expliquer le phénomène par une coïncidence ou une inférence logique de Victor basée sur les informations infraverbales transmises au téléphone par la mère, alors le psychologue ne ferait que plaquer son scepticisme sur ce qui devrait plutôt relever de l'indécidable. Il sortirait effectivement de sa neutralité en prétendant maîtriser un réel qu'il n'a pas investigué. Il serait mieux inspiré d'entendre ce besoin de convaincre dans ce qu'il évoque de narcissique et de fantasmatique au sujet d'un enfant devenu « doré ». Ainsi, quelle enveloppe psychique pour une mère qui pense que son enfant lit dans ses propres pensées ? Comment l'enfant se structure dans cette place où ses dons surpassent ceux de sa mère ?

Car sa mère n'est pas totalement hors de ce champ du paranormal : depuis un an, elle vit des phénomènes paranormaux qu'elle dit pouvoir désormais "gérer». Ces phénomènes sont décrits comme des « sorties du corps traumatisantes » : dans une zone intermédiaire entre le rêve et l'éveil, elle sent son corps qui commence à flotter. Des entités commencent à l'attaquer en se mettant à califourchon sur elle, notamment un petit bonhomme avec un chapeau. Elle reçoit des coups de poing, la lutte est terrible. Elle a l'impression d'avoir la cervelle aspirée, le tout avec un bruit d'ascenseur en marche et parfois des voix peu distinctes. Heureusement, grâce à ses interlocuteurs virtuels, elle a appris à les « bloquer ».

Là encore, le psychologue peut disposer d'un modèle explicatif : celui de la paralysie $d u$ sommeil. Ce trouble, encore méconnu par le grand public, touche environ $25 \%$ de la population sans être lié avec une psychopathologie [8]. Il s'agit, dit grossièrement, d'un courtcircuit hormonal qui fait réveiller l'esprit alors que le corps est encore paralysé. Ce bref passage entre deux états de conscience fait paniquer le cerveau, qui se met à produire des hallucinations sensorielles et psychiques. Mais la tentation de rassurer la mère avec cette explication toute faite peut être dommageable : ne risque-t-on pas de passer à côté des enjeux 
psychiques? En l'occurrence, réduire son vécu à un phénomène individuel viendrait fracturer la dimension interpersonnelle du questionnement. Car la mère dit bien ne plus souffrir de ces phénomènes, relativement contrôlés. Ce qui vient adoucir ces vécus, c'est qu'elle a été confortée dans l'idée qu'elle n'est pas folle grâce à du soutien trouvé sur Internet. De plus, son fils vivant naturellement ses propres dons, toute la problématique a donc glissé sur lui.

Le cours des entretiens amène, comme souvent, à se décentrer des expériences exceptionnelles pour éclairer leur contexte d'apparition. On apprend ainsi que la mère est séparée depuis deux ans de son mari violent et adultérin. Voici quelques mois, celui-ci a piraté son ordinateur découvrant alors ses visites sur des sites ésotériques et ses échanges autour des phénomènes paranormaux. On lui a conseillé de faire de l'écriture automatique pour découvrir quelle est l'entité qui la guide, et de développer ses dons de «magnétisme ». Tout à fait imperméable à ces aspects («Il n’est pas très évolué spirituellement », dira la mère), le père a crû que son ex-femme était tombée dans une secte, entraînant son enfant dans des idées folles, et a déclenché une enquête sociale. Il a également exigé que Victor voie un pédopsychiatre, tout en manipulant ce dernier pour qu'il lui rapporte tout. L'enquête sociale s'est révélée négative. Mais la situation laisse facilement transparaître la tension régnant dans la famille. La mère, humiliée, accusant par ailleurs des échecs socioprofessionnels, a traversé une phase dépressive. La position perverse et antiparanormal du père a alors renforcé l'aspect fusionnel de la relation mère-enfant, tout en divisant le corps social entre ceux à qui on peut parler de paranormal et ceux à qui on ne peut pas.

Victor subit ce divorce et cette pression sans trop s'en plaindre, comme pour protéger une unité familiale. Ses dons de guérison peuvent s'inscrire dans ce désir d'être un palliatif, tout comme la télépathie vient faire le lien entre des personnes distantes mais affectivement liées. Or, toute cette problématique de la paranormalité dans le fonctionnement psychique de la famille ne peut être entendue nulle part. Victor a «vu» qu'il ne pouvait rien en dire au 
pédopsychiatre, qui s'est contenté d'un entretien pour dire que tout allait bien chez cet enfant. Victor et sa mère partagent une même façade factice devant les enquêtrices sociales, mettant en avant le problème manifeste des tensions suite au divorce. Depuis peu, Victor va voir une psychologue à qui il confie seulement - mais au moins - ses ressentis de la violence de son père. Il faut dire que celui-ci lui a dit : «Ta mère est folle, dis lui d'aller voir un psy ! », ce qui engage Victor dans un abord biaisé de la consultation psychologique.

C'est ainsi que le circuit médico-psychologique traditionnel peut passer à côté d'une portion de la vie psychique qui ne vient pas se déposer dans n'importe quel dispositif. Et pourtant, ces influences culturelles et interpersonnelles constituent une donnée capable de faire vaciller un diagnostic. En effet, si Victor parle enfin de ses contacts télépathiques, devrait-on diagnostiquer une hallucination du côté de la psychose? L'histoire de Victor montre bien comment cette représentation de «l'enfant doré », même présentée dans un microire et un mi-dire, peut venir combler une dissolution familiale, tout en soutenant l'enfant dans une construction ambivalente de la place qui lui revient.

\section{Une marginalité tentaculaire}

Les mouvements indigo s'ancrent en général dans un syncrétisme New-Age. Toutes les thématiques s'y retrouvent [18]: auras, travail énergétique, gymnastique du cerveau, anges, fleurs de Bach, cristaux, alimentation naturelle, homéopathie... Cette marginalité est souvent mise en avant, afin de ridiculiser les doctrines indigo. Mais le syncrétisme atteint par ces mouvements dépasse encore ce cadre pour conquérir des zones frontières, où des controverses laissent l'opinion publique dans une indécision. Les tenants des enfants indigo s'emparent de ces débats, de telle façon que les frontières culturelles deviennent troubles, reflétant l'ambigüité de la définition d'une secte dans son opposition à une nouvelle 
spiritualité. Capables de réinterpréter toute l'histoire des rapports de la jeunesse avec l'occultisme, les mouvements indigo proposent un système alternatif, aux aspects totalitaires. Il y a en particulier un chantage au suicide qui piège les parents inquiets : si ceux-ci ne tiennent pas compte du destin particulier de leurs enfants, ils devraient se sentir coupables de leur malheur, voire de leur suicide. Ce genre d'emprise négative va de paire avec des réflexions holistes riches d'espérances, sur le système pédagogique, sur l'écologie, sur les évolutions de la spiritualité, sur la politique, sur la psychopathologie et en particulier sur la science, par le recours aux disciplines marginales de la parapsychologie et de l'ufologie (étude des Objets Volants Non Identifiés).

Cette multiplicité ouvre à des dimensions qui débordent le simple cadre de la croyance naïve, et touchent en réalité aux problèmes modernes du lien social. Ainsi, les enfants indigo sont associés aux rencontres rapprochées avec les extraterrestres et aux récits d'enlèvements. Aux États-Unis, une importante littérature a popularisé ces thèmes, provenant même de cliniciens reconnus comme le psychiatre d'Harvard John Mack (Pour une critique, voir [25]). Une nouvelle théorie de l'origine s'est répandue : le génome des enfants indigo aurait été modifié par les extraterrestres. Cette idée n'est pas psychotique en elle-même, car elle peut convenir à une constitution névrotique sous forme d'un roman familial, ou à une psychose recréant une filiation symbolique. Il faut donc entrer dans le détail de ce questionnement de l'origine, et écouter les modalités du lien qui sont en jeu. Ainsi, l'intervention des extraterrestres peut avoir lieu à différents niveaux : soit en amont en «contactant » les parents et modifiant par bio-ingénierie leur future procréation ; soit en aval en manipulant la structure de filiation, choisissant les parents en fonction du potentiel de l'enfant ; ou encore en enlevant l'enfant et en le « retouchant ».

Aussi incroyables qu'elles puissent paraître, ces explications donnent un sens à différentes angoisses de parents, quitte à les faire se courber d'admiration devant leur propre 
progéniture. L'indigo est présenté comme un état, pas comme un défaut. «Nul n'en est responsable : ni les enfants, ni les parents (qui sont enfin rassurés). Il est donc tout à fait inutile de vouloir changer ces enfants, il va falloir s'y faire et les adultes devront agir en conséquence, en faisant preuve de douceur, de respect, d'amour, de compréhension et de tolérance. Le parent qui aura su devenir le complice de son enfant découvrira alors des joies insoupçonnées et le bonheur lui est promis. » [31, p. 139]. Cette inversion des rôles entraîne une autre gestion des responsabilités éducatives, conduisant à suivre les préceptes et les cours bien encadrés par les lucratives «écoles spécialisées » des tenants de l'indigo, lesquels promeuvent une nouvelle codification du métier impossible de parent.

L'un des introducteurs des enfants indigo, James Twyman, centre plutôt la problématique autour de l'existence de capacités «psi » (c'est-à-dire «parapsychologiques ») chez les enfants. Il a participé à la mise en scène du film Indigo (2003), vend des livres et des cours par Internet basés sur cette question, tient des conférences au prix de 300 dollars US par adulte [1]. Il fait explicitement référence au prestidigitateur charismatique Uri Geller (1946-) qui revendique, depuis les années 1970, le pouvoir de plier des petites cuillères ou d'arrêter des montres seulement par la pensée. On pénètre alors dans le champ controversé de la parapsychologie, dont il est difficile de reconstituer le fond de réalité. Ce prestidigitateur israélien est devenu célèbre dans le monde entier suite à sa participation à des expériences prétendument scientifiques (notamment publiées dans Nature), au cours de ses nombreux déplacements généralement accompagnés de performances grotesques sur les plateaux télé. Le livre qui lui est consacré par le Dr Andrija Puharich [29] a participé à la diffusion d'une mythologie autour de cet icône d'un genre nouveau, vivant des expériences exceptionnelles sur fond de politique inter-galactique. C'est un héritage que revendiquent tout à fait les tenants des enfants indigo, Colin Wilson [32, p. 309] rapportant par exemple que Puharich travaillait, à la fin de sa vie, sur les « enfants supranormaux » qui semblaient proliférer. Les 
«thérapeutes indigo » tentent par ailleurs de recréer un dispositif qu'ils jugent apte à faire émerger les facultés parapsychologiques latentes [5], à l'image du Mouvement pour le Potentiel Humain des années 1960.

Avant même les enfants indigo, les prestations d'Uri Geller ont déclenché un phénomène sociologique mondial parfois comparé à une épidémie [2]. Une cohorte d'adolescents s'est présentée aux portes des studios télés et des instituts scientifiques, prétendant être doués des mêmes pouvoirs que Geller. Dans les comptes-rendus des parapsychologues, on aperçoit par endroits le rôle des parents. Ils sont parfois à l'origine de la démarche des «mini-gellers », désirant reconnaître certaines capacités à leurs adolescents et les conduisant vers les spécialistes présumés. Ils peuvent aussi assister à certaines expériences, ou s'y opposer. Ils tentent parfois de retirer l'enfant des mains des expérimentateurs à qui ils les avaient confiés, montrant un investissement ambivalent de l'image de «l'adolescent psychokinète ». Le contexte de l'époque semble aujourd'hui dépassé, mais ses conditions d'apparition pourraient ressurgir. L'icône d'Uri Geller a agi à la manière d'un agent épidémique. Peu importe la réalité de ses propres pouvoirs, ils ont néanmoins actionné un ressort dans l'imaginaire social au niveau international. Si des adultes ont également réagi en affirmant posséder les facultés occultes de Geller, le phénomène s'est tout de même concentré sur la gent adolescente. Or, il ne s'agit pas d'une pure identification,

car l'histoire de Geller ne laisse rien transparaître d'un rapport de l'adolescence à l'occultisme. Sa jeunesse est tout à fait normale, et ses premières prestations d'illusionniste ont lieu après qu'il ait atteint la vingtaine. C'est donc que cette mythologie est venue alimenter des fantasmes déjà présents dans la culture.

\section{L'adolescent mutant dans la culture}


«La mue est un état de maladie normale » dans lequel serait plongé l'adolescent, disait Dolto [12, p. 113]. En plus de brouiller les cartes des diagnostics, la métaphore d'un adolescent mutant introduit une possibilité d'être surpris par une évolution pourtant annoncée. L'étude historique des rapports entre l'adolescence et l'occultisme [13] fait également ressortir cette configuration imaginaire particulière. Le jeune serait porteur de nouvelles capacités, inscrites sur le plan biologique mais également associées à une forte spiritualité. Il incarnerait ainsi un progrès de l'humanité, un «joker de la création » [6] selon Nancy Ann Tappe, créatrice du concept d'enfant indigo. L'adolescent mutant se révèlerait lors de sa crise d'adolescence, désorientant ses parents et toutes les institutions. Ce sont Les enfants d'Icare (1953) du romancier Arthur C. Clarke, les X-Men de Marvel Comics (depuis 1963), la série télé des Tomorrow People (1972-2007), l'incontrôlable Carrie (1974) du romancier Stephen King, et la possédée de L'exorciste (1973), les superhumains de Suberboy (1988-1992) et Smallville (depuis 2001), Sabrina l'apprenti sorcière (depuis 1996), Kyle X.Y. (depuis 2006), etc. Les exemples littéraires sont nombreux : une grande portion des mangas adopte le thème récurrent de l'enfant mutant, Harry Potter raconte la vie d'élèves sorciers (depuis 1998), et dans Phaenomen (depuis 2006) ces enfants doués doivent s'échapper de l'enceinte psychiatrique où on les enferme.

On peut schématiser cette configuration imaginaire par ses trois axes privilégiés :

- L'opposition d'un monde visible et d'un monde invisible : les capacités en question agissent comme un différenciateur. Elles ne touchent qu'une partie minoritaire des humains, ce qui permet de distinguer ceux qui n'en sont pas de ceux qui en sont (Par exemple, «Moldus » et « Sorciers » dans Harry Potter). Ces derniers ont alors accès à un monde resté invisible ou voilé aux yeux des autres.

- L'opposition entre «breaking down » et «breaking out» : la crise d'adolescence est considérée comme une superposition de deux devenirs possibles. Il y a à la fois 
l'aspect pathologique (breaking down comme rupture du développement normal), et l'aspect créatif et libérateur (breaking out comme évasion ou émergence). Souvent, le regard porté par les agents du monde visible ramènera tout à l'aspect pathologique, tandis qu'une minorité défendra l'aspect créatif et libérateur. La dynamique d'opposition de deux mondes sera renforcée par ce conflit sur la description de la crise. La question du diagnostic différentiel est fortement sollicitée.

- Les nouvelles transgressions et les nouvelles limites: Ce qui donne son aspect «mutant» à cette configuration, et ce par quoi le paranormal est introduit, reste la possibilité de nouvelles transgressions. Elles impliquent alors un ancrage dans le corps d'un signe de la mutation (absence de nombril de Kyle X.Y., cicatrice en forme d'éclair d'Harry Potter, anormalité génétique ou physiologique, etc.), comme si une mutation purement psychologique responsabiliserait trop l'individu, d'où le besoin d'une castration venant limiter une jouissance débridée. Forces et faiblesses s'entremêlent.

Au final, ces structures imaginaires constituent une forme typique rencontrée lors du passage adolescent. Selon l'analyse de Pascal Le Maléfan [23], ces mutants incarnent des personnages littéralement Autres que le commun, dotés de pouvoirs supposant un monde gouverné par d'autres lois, et qui possèdent ce supplément d'âme que l'adolescent entend recouvrer. Par ce fantasme d'Autres mythologiques, l'adolescent reste fasciné par un impossible qui ne l'est pas tant que cela, et qui indiquerait que la castration pourrait être contrebalancée. Face à la chute de la croyance infantile en l'incarnation imaginaire de l'Autre, le sujet en adolescence a donc toujours la possibilité de créer des Autres mythologiques. Ceux-ci vont pouvoir soutenir une opération adolescente où flambe l'imaginaire, en même temps que l'épreuve de réalité vacille par moment.

L'imagerie du mutant dans les cliniques adolescentes serait l'effet de la conjonction entre des mouvements psychiques et de nouvelles références sociales. Notre société 
postmoderne prétend, grâce aux progrès de la science et des technologies, se passer des dieux. Mais ceux-ci continueraient à hanter le psychisme humain. «Comment alors le sujet, toujours soumis à ces mâttres de l'infantile, fait-il face à l'absence des tuteurs divins, de la référence ? » [24, p. 15]. La première façon classique de se débrouiller est de remplacer le « dieu » paternel de l'enfance par des références sociales : professeurs, mâttres, leaders, etc. Mais, selon Lesourd [24, p. 16], il y aurait depuis 1970 des formes nouvelles de création d'idole qui ont pris le dessus dans la réalité de notre lien social. «En faisant de l'Homme la référence, le nouveau lien social a libéré l'humain de la culpabilité qui était le limon de toutes les religions, et il a mis en avant l'autre figure idéale de l'homme, celle dans laquelle il se prend lui-même pour un dieu, le moi-idéal issu de la toute-puissance hallucinatoire infantile, si bien décrite par la psychanalyse anglo-saxonne. C'est ainsi que, au temps du nécessaire désenchantement pubertaire des dieux infantiles, les adolescents se vouent à la passion mystique du nouveau dieu social : l'homme, c'est-à-dire eux-mêmes. » [24, p. 19]. Il n'est donc pas anodin que les thèmes des facultés latentes ou du «potentiel humain » croisent ceux de la latence et du devenir adulte.

\section{Conclusion : comment ne pas être un Moldu}

La question des enfants indigo ne relève pas seulement d'une réflexion anthropologique: elle pénètre aussi dans le champ clinique. Ainsi, Jeanne Siaud-Facchin, psychologue clinicienne travaillant sur les surdoués ne compte plus les questions portant sur les enfants indigo lors de ses conférences [9]. Les interrogations fusent également sur les 
forums Internet, et dans un circuit médico-psychologique parallèle. Même s'il s'agit encore d'une marge de la psychopathologie, il est dommageable de ne pas l'aborder pour les raisons déjà avancées ci-dessus. La question se pose aussi du type de discours à tenir : faut-il simplement dénoncer, comme le font déjà les missions de lutte anti-sectes, les abus autour de ces mouvements New Age ? Faut-il se positionner sur le plan scientifique et rejeter en bloc la preuve de l'existence d'enfants indigo munis d'auras violacées et d'ADN modifié ? Ne faut-il être que clinicien et attendre le prochain patient indigo pour écouter toute la singularité de sa problématique ?

Notre option a été de ne pas nous focaliser sur un aspect du phénomène, qui lui-même est protéiforme ainsi qu'on a pu le constater. Il s'agit de s'ouvrir à un maximum de dimensions, couplant psychopathologie et anthropologie clinique. Cette bienveillance permet de n'être pas un simple « Moldu », pour utiliser le vocable de la saga Harry Potter et repris à leur compte par certains protagonistes [21], désignant ceux qui ignorent et stigmatisent de fait la magie. Ici, il s'agit d'accepter l'existence d'enfants indigo, à la fois comme effets d'une catégorie artificiellement construite que comme être humains incarnant ces catégories. On se place alors dans la lignée des épistémologies constructivistes d'une redoutable efficacité dans les sciences sociales et humaines [26, 4, 17]. Les croyances et promesses porteuses de ces mouvements ne doivent pas être réduites immédiatement par une analyse critique basée sur des préjugés. En réalité, les tenants de ces mouvances ne sont pas les seuls responsables de la niche écologique où elles prospèrent.

Faisant des mouvements indigo un véritable courant culturel, bien que minoritaire et marginal, on se met enfin à portée des problématiques et des références dans lesquelles sont plongées de nombreuses personnes. Les psychologues ne doivent plus être «les grands absents de ce débat » comme l'indiquait très justement Claudie Voisenat en 2005 [31, p. 159]. En suivant les préoccupations de la clinique, on évite une vision bipolaire du phénomène qui 
entrave les possibilités d'écoute et de soin. Mais à cette bienveillance, il faut adjoindre toute la rigueur possible pour ne pas se faire soi-même le promoteur de ces mythes parasites. Il faut ainsi se donner les moyens de décrypter la fascination sociale pour l'adolescent mutant, tout en ajustant le mieux possible les outils de diagnostic différentiel pour n'oublier aucune dimension de l'expérience humaine.

\section{Références :}

1. Anderson L. Indigo : la couleur de l'argent. Les enfants indigo [En ligne]. 2003. Disponible sur: <http://www.charlatans.info/indigo.shtml > (consulté le 19.09.2009)

2. Bender H. Zur Uri Geller-Epidemie, Zeitschrift für Parapsychologie und Grenzgebiete der Psychologie $1974 ; 16$ : 43-45.

3. Bon N. Les enfants indigo [En ligne]. 2004. Disponible sur: <http://www.freudlacan.com/articles/article.php?url_article=nbon041204> (consulté le 19.09.2009)

4. Borch-Jacobsen M. Folies à plusieurs: De l'hystérie à la dépression, Paris, Les Empêcheurs de Penser en Rond, 2002.

5. Boylan R. Star Kids Benefit From Special Schooling [En ligne], Academy of Clinical Close Encounter Therapists (ACCET) Newsletter 1999. Disponible sur: <http://inspiration.ifrance.com/inspiration/_private/enfants.indigos.htm> (consulté le 19.09.2009)

6. Carroll L, Tober J. Les enfants indigo : enfants $d u 3^{e}$ millénaire, Outremont, Ariane Editions, 1999.

7. Carroll L, Tober J. Célébration des enfants indigo, Outremont, Ariane Editions, 2002.

8. Cheyne JA. Sleep paralysis episode frequency and number, types, and structure of Associated Hallucinations, Journal of Sleep Research 2005, 14 : 319-324. 
9. Chupin J. Enfants indigo: les nouveaux missionnaires [En ligne]. Le Monde de l'Education, $\quad 1^{\mathrm{er}} \quad$ novembre $2004 . \quad$ Disponible sur : <http://appy.ecole.free.fr/articles/20041100d.htm> (consulté le 19.09.2009)

10. Clarke AC. Childhood's end (Les enfants d'Icare), New York, Ballantine Books, 1953.

11. Corcombet M, Denoyer F, Laurent G, Mansour M. Les enfants indigo II, Dossier d'étudiants du cours de Zététique \& Approche scientifique du paranormal, Université Joseph Fourier de Grenoble, 2007.

12. Dolto F. La cause des adolescents, Paris, Robert Laffont, 1988.

13. Evrard R. Adolescence et occultisme: mise en perspective différentielle, Mémoire de Master 2 Recherche «Psychopathologie et Etudes Psychanalytiques » non publié, Université Louis-Pasteur de Strasbourg, 2008.

14. Goldstein J.. Consoler et classifier : L'essor de la psychiatrie française, Paris, Institut Synthélabo, coll. « Les Empêcheurs de Penser en Rond », 1997.

15. Gori R, Del Volgo M-J. Exilés de l'intime : La médecine et la psychiatrie au service du nouvel ordre économique, Paris, Denoël, coll. «Médiations », Série «L'espace analytique », 2008.

16. Hacking I. L’âme réécrite: Essai sur la personnalité multiple et les troubles de la mémoire, Paris, Institut Synthélabo, coll. «Les Empêcheurs de penser en rond », 1998.

17. Hacking I. Les fous voyageurs, Paris, Les Empêcheurs de penser en rond/Le Seuil, 2002.

18. Hehenkamp C. Vivre avec un enfant indigo: Conseils et exercices pour une relation détendue, Paris, Exergue, 2004.

19. Jeannaud H. Le marché des «enfants indigo » [En ligne]. Journal du dimanche du 28 décembre 2003.

Disponible

sur :

<http://www.psyvig.com/default_page.php?menu=1010\&page=4> (consulté le 19.09.2009) 
20. Kemp D. Le Syndrome de l'enfant Téflon, Montréal, Éditions E=MC2, 1988.

21. Leland J. Are they here to save the world? [En ligne] New York Times, 12 janvier 2006. Disponible sur : <http://www.nytimes.com/2006/01/12/fashion/thursdaystyles/12INDIGO.html> (consulté le 19.09.2009)

22. Le Maléfan P. Folie et spiritisme Histoire du discours psychopathologique sur la pratique du spiritisme, ses abords et ses avatars, Paris, L'Harmattan, coll. «Psychologiques », 1999.

23. Le Maléfan P. «La vérité est ailleurs »: La place du paranormal à l'adolescence comme mode de traitement du réel pubertaire, Adolescence 2008, 26(3) : 709-721.

24. Lesourd S. L'incontournable passion mystique de l'adolescent, Adolescence 2008, 26(1) : $9-21$.

25. Maleval J-C. Une épidémie américaine. Le syndrome d'enlèvement extra-terrestre, In : Collectif, Le conciliabule d'Angers. Effets de surprise dans les psychoses, Agalma-Le Seuil, 1997.

26. Mancini S. (dir.) La fabrication du psychisme: Pratiques rituelles au carrefour des sciences humaines et des sciences de la vie, Paris, La Découverte, coll. « Recherches », 2006.

27. Nathan T. (dir.) L'enfant ancêtre, Paris, La pensée sauvage, coll. «Bibliothèque d'ethnopsychiatrie », 2000.

28. Pignarre P. Comment la dépression est devenue une épidémie, Paris, La Découverte, 2001.

29. Puharich A. Uri Geller, Paris, Flammarion, 1974.

30. Swanson JM, Sergeant JA, Taylor E, Sonuga-Barke EJS, Jensen PS, Cantwell DP. Attention-deficit hyperactivity disorder and hyperkinetic disorder, Lancet 1998, 35 : 429-433. 
31. Voisenat C, Lagrange P. L'ésotérisme contemporain et ses lecteurs. Entre savoirs, croyances et fictions, Paris, BPI/Centre Pompidou, coll. «Études et recherche », 2005.

32. Wilson C. Alien Dawn : An Investigation into the Contact Experience, Londres, Virgin Publishing, 1988. 


\title{
Une marge de la psychopathologie contemporaine : les « enfants indigo »
}

\author{
Par Renaud Evrard* et Pascal Le Maléfan**
}

* Renaud EVRARD, psychologue, doctorant en psychologie, Université de Rouen. 2 place Galilée, 67380 Lingolsheim. Contact pour la relecture des épreuves : renaud.evrard@etu.univ-rouen.fr ; 0671188186.

** Pascal LE MALEFAN, docteur en psychologie, psychanalyste, Professeur de psychopathologie, Université de Rouen, 17, Rue Lavoisier F-76821 Mt-St-Aignan cedex.

\section{Résumé :}

Les «enfants indigo » est le nom générique donné par un ensemble de mouvements New Age à des enfants considérés comme «surhumains » et qui se présentent généralement avec des difficultés censées découler de l'inadaptation de leur environnement à leur supposé haut potentiel. En gestation depuis les années 1970, le mouvement se répand dans les pays francophones depuis 1999. Cet article propose une description générale des conditions d'émergence et de développement des mouvements autour des «enfants indigo », de façon à situer leur «niche écologique». Cette description sera utile aux approches psychopathologiques infanto-juvéniles car ces références culturelles pénètrent le champ de la santé mentale, ainsi que l'illustrera le cas clinique de Victor.

\begin{abstract}
:
The "indigo children" is the generic name given by some New Age movements to purposely "superhuman" children who have generally problems which arise from the inadequacy of their environment to their supposed high potential. In gestation since the 1970s, the movement is spreading in French-speaking countries since 1999. This article offers a description of the conditions of emergence and development of movements around the "indigo children" in order to seize their "ecological niche". This will be useful for children and adolescents psychopathological approaches because these cultural references enter the mental health field, as illustrated by the clinical case of Victor.
\end{abstract}

Mots-clés : Marges de la psychopathologie - Trouble de déficit de l'attention / hyperactivité - enfants indigo - Culture adolescente - Constructivisme des maladies mentales Clinique des expériences exceptionnelles 